\title{
Laparoscopic treatment of giant solitary hepatic cyst in a geriatric patient
}

\author{
S Ricciardi*, R Giansini, E Mion, P Mainente, S Faccin and L De Santis
}

Address: U.O.A. Di Chirurgia Generale, Ospedale "San Lorenzo" Valdagno - VI, Italy

* Corresponding author

from XXI Annual Meeting of The Italian Society of Geriatric Surgery

Terni, Italy. 4-6 December 2008

Published: I April 2009

BMC Geriatrics 2009, 9(Suppl I):A45 doi: 10.1 186/147I-23I8-9-SI-A45

This abstract is available from: http://www.biomedcentral.com/I47I-23 I8/9/SI/A45

(C) 2009 Ricciardi et al; licensee BioMed Central Ltd.

\section{Objective}

Solitary congenital liver cysts are a rare finding and present a challenge in the diagnosis. The role of laparoscopy surgery in the management of benign cysts is now the gold standard for treating selected, giant, accessible, symptomatic or complicated cysts. Our objective was to assess the feasibility, the safety and the efficacy of the minimally invasive technique procedure for treating giant hepatic cyst even in geriatric patient.

\section{Methods}

In support of the laparoscopic approach in the elderly, we here describe our minimally invasive technique in a patient with an isolated cystic mass located in the right lobe of the liver, in the anteroinfeior (V-VI) and posteriosuperior (VI-VIII) segments (Figure 1). A 65-year-old woman reported progressive upper abdominal distension, and digestive complaints that are unexplained by other findings. Our case was initially diagnosed by ultrasound (solitary cyst, unilocular, spherical shape, smoothwalled, and absence of internal echoes, measuring $15 \mathrm{~cm}$ in diameter) and confirmed by CT scan and MRI, which also gave further information regarding the extent of the mass and its proximity to any vital structures within the liver. Serological tests for hydatid liver disease were negative and an upper gastrointestinal endoscopy was normal. Alkaline phospatise and the total bilirubin level were mildly elevated. Thereafter the patient was scheduled for laparoscopic cyst excision. After trocar placement, similar to that for laparoscopic cholecistectomy, a small opening was made in the cyst and the contents aspirated for cytology and culture. Using a monopolar hooked cautery, the cyst was resected free from the liver surface, followed by an omentoplasty into the remaining cyst cavity (to prevent closing roof defect and cyst recurrence). The cyst was not in communication with biliary tree and not biliary leak has been reported. The procedure was completed laparoscopically without intraoperative complication and no need for blood transfusion. The operative time was 240 minutes.

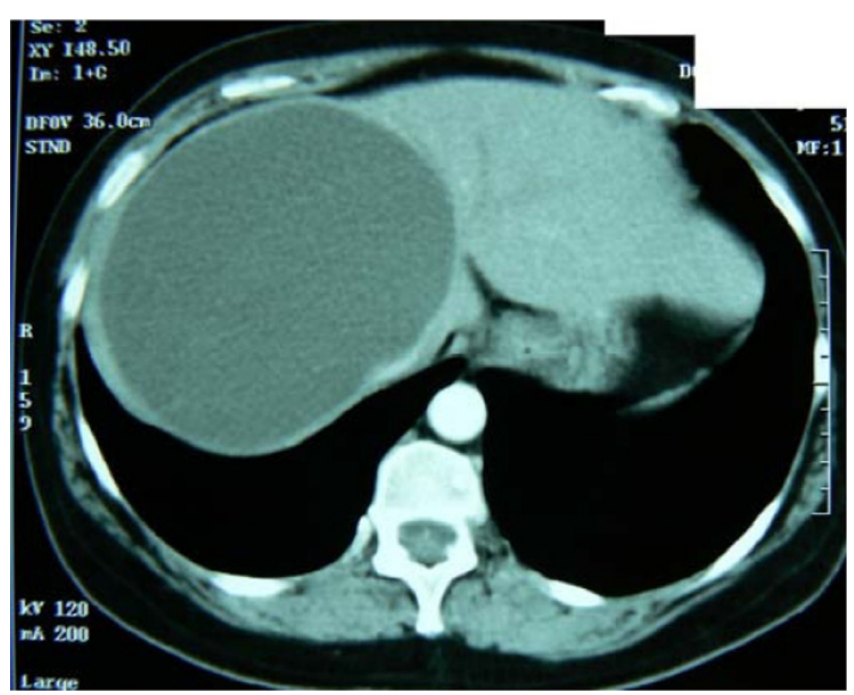

Figure I

CT scan: appearance of a large hepatic cyst. 


\section{Results}

The cyst has been resected free from the liver surface without intraoperative complication and no need for blood transfusion. Histopathologic analysis demonstrated a true liver cyst (smooth cyst wall without calcification). Cyst serous fluid sent for CA testing was negative as a tumoral marker. In the early postoperative the patient developed a bilateral pleural effusion, treated successfully conservatively. The postoperative hospital stay was 12 days.

The patient is alive and free of symptoms during a followup of 12 months, without any signs of cyst recurrence.

\section{Discussion}

The precise frequency of liver cysts is difficult to estimate, because most do not cause symptoms and require no treatment. When cysts become large and cause symptom treatment is warranted. No medical therapy has proven curative. From technical point of view cyst may be treated by percutaneous aspiration under ultrasound or CT guidance in combination with sclerotherapy with alcohol or other agents. However the cyst fluid is continually secreted by the epithelial lining of the cyst and for this reason, needle aspiration of simple cyst is not curative and it may cause bleeding and the development of hepatic abscess do to contamination of the cavity that should become a problem difficult to resolve. Today, the general agreement is that laparoscopic unroofing of large simple cyst (the so called Lin procedure) offers the best balance between efficacy and safety.

In our experience we preferred remove completely the wall of the cyst, to avoid cyst recurrence, by suppressing further fluid secretion. However, excision of the entire cyst wall is not necessary if is possible ablate the remaining epithelium with elettrocautery or an argon beam coagulator. Surgical cyst fenestration can be done laparoscopically in elderly achieving at least similar results to those offered by an open approach, and is accompanied by the usual postoperative benefits of laparoscopic surgery.

\section{Conclusion}

Hepatic symptomatic giant cysts are suitable for the laparoscopic approach even in elderly patients. In selected patients total excision of the cyst is safe, associated with minimal morbidity and good long-term outcome and offers all the advantages of minimally invasive surgery.

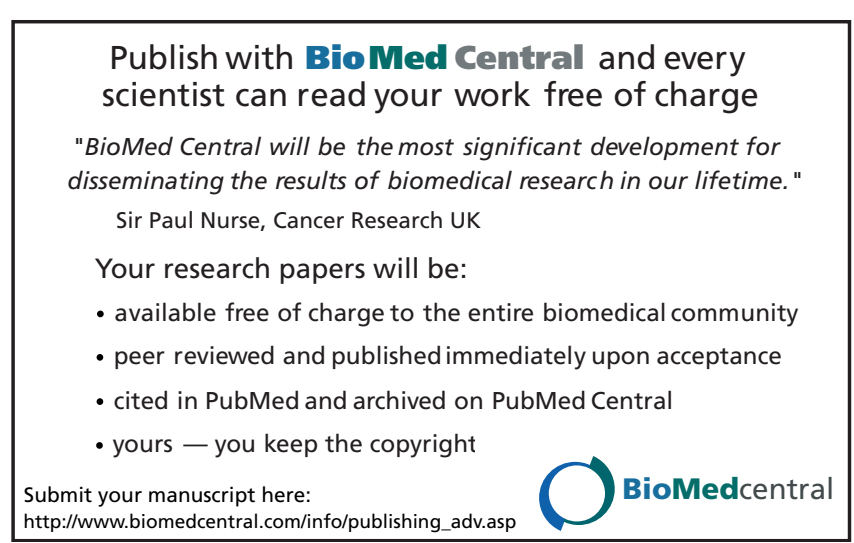

Page 2 of 2

(page number not for citation purposes) 